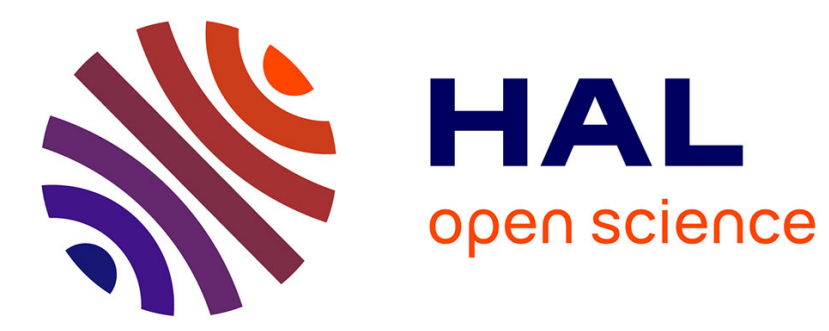

\title{
Molecular basis for fluidization of cancer cells
}

René Marc Mège

\section{To cite this version:}

René Marc Mège. Molecular basis for fluidization of cancer cells. Nature Materials, 2019, 18 (11), pp.1147-1148. 10.1038/s41563-019-0521-2 . hal-03040837

\section{HAL Id: hal-03040837 https://hal.science/hal-03040837}

Submitted on 21 Dec 2020

HAL is a multi-disciplinary open access archive for the deposit and dissemination of scientific research documents, whether they are published or not. The documents may come from teaching and research institutions in France or abroad, or from public or private research centers.
L'archive ouverte pluridisciplinaire HAL, est destinée au dépôt et à la diffusion de documents scientifiques de niveau recherche, publiés ou non, émanant des établissements d'enseignement et de recherche français ou étrangers, des laboratoires publics ou privés. 


\section{Cell invasion: Molecular basis for fluidisation of cancer cells}

A molecular pathway has been identified in the regulation of unjamming to overcome cancer cell migration and proliferation arrest leading to collective cell invasion.

\section{René Marc Mege}

Institut Jacque Monod, Université Paris Diderot, Paris, France

Email: Rene-marc.mege@ijm.fr

Cells in humans and animals can flow locally like a fluid while maintaining mechanical integrity, similarly to inert soft matter such as colloidal suspensions. Moreover, cell collectives in vitro and in vivo have been shown to display liquid-to-solid phase transition. Researchers have utilised basic concepts in solid matter such as glassy dynamics and jamming to investigate the behaviour of living tissues. The jamming transition is characterized by an abrupt kinetic arrest. It can be induced by steric hindrance upon a particle density threshold preventing the particles to explore the space. The critical jamming parameters for a system also involve particle shape, deformability and antiparticle forces. During tissue growth cells are free to move, as in a fluid, but their motion becomes constrained as density increases. At a critical density motility ceases and collectives solidify undergoing jamming transition [1, 2]. Palamidessi and colleagues now report in Nature Materials on the unjamming in normal and tumorigenic 2D epithelial sheets and 3D spheroids [a].

The existence of a jamming transition in the embryo, in 3D, has been recently shown to be essential for vertebrate body axis elongation [3]. The reverse solid-to-liquid (unjamming) transition might represent a complementary gateway to epithelial cell migration, enabling mature tissues to flow [4]. Indeed abnormal unjamming in 2D epithelial layers in culture has been associated to asthma pathobiology [5] and tumor dissemination [6]. Cellular jamming differs from jamming of inert matter in that cells are active particles that actively change shape, cortical tension, intercellular adhesion and motility [1, 2], rendering the system much more complex and thus, how cells control the jamming/unjamming transition remains puzzling.

Previously, Giorgio Scita and colleagues showed that the overexpression of the small GTPase RAB5A, a master regulator of early endosomes, was sufficient to reawaken the motility of jammed 2D monolayers of human mammary epithelial cells [6]. By enhancing endosomal trafficking and cell surface molecules internalization, RAB5A impacts on junctional tension, topology and dynamics of the adhesion molecule E-cadherin, facilitating neighbours exchange and finally large scale, coordinated motility over tens of cells in otherwise kinetically arrested cells. This was associated with increased traction forces applied by the cells on the extracellular matrix and to the extension of Rac1-driven lamellipodial cell protrusions, which align with local tissular velocity vectors. These authors proposed a model in which both changes in junctional tension and active cell reorientation regulated by RAB5Adependent endosomal trafficking determine regimes of monolayer dynamics that explain reawakening of locomotion and local unjamming. They propose that these changes in multicellular dynamics may be exploited by tumours for invasion. However, the biomolecular machinery behind unjamming and its pathophysiological relevance remained fully unclear with no data available on the molecular mechanisms linking RAB5A trafficking, intercellular dynamics and actin-based protrusion. Moreover, there are neither reports on the effects of this unjamming in 3D nor demonstration of an implications in tumoral invasion. 
Palamidessi and colleagues show that increased levels of RAB5A promote unconventional, nonclathrin-dependent internalization of Epidermal Growth Factor Receptor (EGFR) leading to the activation of the signalling kinase ERK1/2 and phosphorylation of the actin nucleation activator WAVE2. This cascade stimulates lamellipodia extension and triggers collective motility. The researchers thus identified part of the signalling cascade underlying the previously reported effect of RAB5A expression on the fluidization of jammed epithelial monolayers. Furthermore, they nicely extend their observations to $3 D$ providing the first evidence that such processes may directly participate in tumor invasion. Specifically, they demonstrate that RAB5A expressing tumor spheroids unjamming at their periphery and that this fluidization is accompanied by persistent and coordinated rotations. The increase strain/stress exerted by the migrating cells on the surrounding extracellular matrix remodels the collagen fibrils facilitating the formation of fiber tracks in which cells collectively migrate from the tumor. These data thus demonstrate that trafficking-induced fluidization of breast tumor cells is sufficient to reawaken motility and promote collective invasion. Unexpectedly, working in $3 D$ on acini of differentiated cells, the authors discovered RAB5A expression also reawakened proliferation in an ERK1/2 pathway.

In conclusion, this study indicates that ERK1/2 activation by RAB5A-mediated endocytosis of EGFR is a key molecular pathway to the unjamming transition, consistently with the emerging role of membrane trafficking in regulating cell migration plasticity and the mechanics of cell-cell interaction. Moreover, the authors show that RAB5A expression is upregulated in invasive foci of human ductal carcinoma in situ and correlates with worse disease-free survival across a variety of breast cancers subtypes, indicating that this pathway is of major importance for the development of breast carcinoma.

There are however some unexplained issues that will need to be addressed in the future such as the link between EGFR/ERK1/2-dependent stimulation of actin nucleation and the previously reported increased dynamics of junctional E-cadherin. Similarly, it is not clear what mediates the changes in junctional tension reported previously [3]. It will be important to address the contribution of EGFRERK1/2-WAVE-dependent actin polymerization on these two biophysical parameters contributing to the jamming-unjamming transition.

This work also raises a very interesting question related to the control of cell proliferation and its coupling with cell migration upon increased endosomal trafficking. Data accumulated in a different cellular model highlight a functional cross-talk between EGFR endocytosis, recruitment of junctional E-cadherin and junctional tension under the control of Merlin/NF2 [8]. Interestingly, these authors also establish a functional link between this pathway and cell proliferation. This track should be now investigated.

\section{References}

1. Park, J.A., et al., Collective migration and cell jamming in asthma, cancer and development. J Cell Sci, 2016. 129(18): p. 3375-83.

2. Garcia, S., et al., Physics of active jamming during collective cellular motion in a monolayer. Proc Natl Acad Sci U S A, 2015. 112(50): p. 15314-9. 
[a] Palamidessi et al. Unjamming overcomes kinetic and proliferation arrest in terminally differentiated cells and promotes collective motility of carcinoma. Nature Materials. https://doi.org/10.1038/s41563-019-0425-1

3. Mongera, A., et al., A fluid-to-solid jamming transition underlies vertebrate body axis elongation. Nature, 2018. 561(7723): p. 401-405.

4. Oswald, L., et al., Jamming transitions in cancer. J Phys D Appl Phys, 2017. 50(48): p. 483001.

5. Park, J.-A., et al., Unjamming and cell shape in the asthmatic airway epithelium. Nature Materials, 2015. 14: p. 1040.

6. Malinverno, C., et al., Endocytic reawakening of motility in jammed epithelia. Nat Mater, 2017.

7. Lecuit, T. and A.S. Yap, E-cadherin junctions as active mechanical integrators in tissue dynamics. Nat Cell Biol, 2015. 17(5): p. 533-9.

8. Chiasson-MacKenzie, C., et al., NF2/Merlin mediates contact-dependent inhibition of EGFR mobility and internalization via cortical actomyosin. J Cell Biol, 2015. 211(2): p. 391-405.

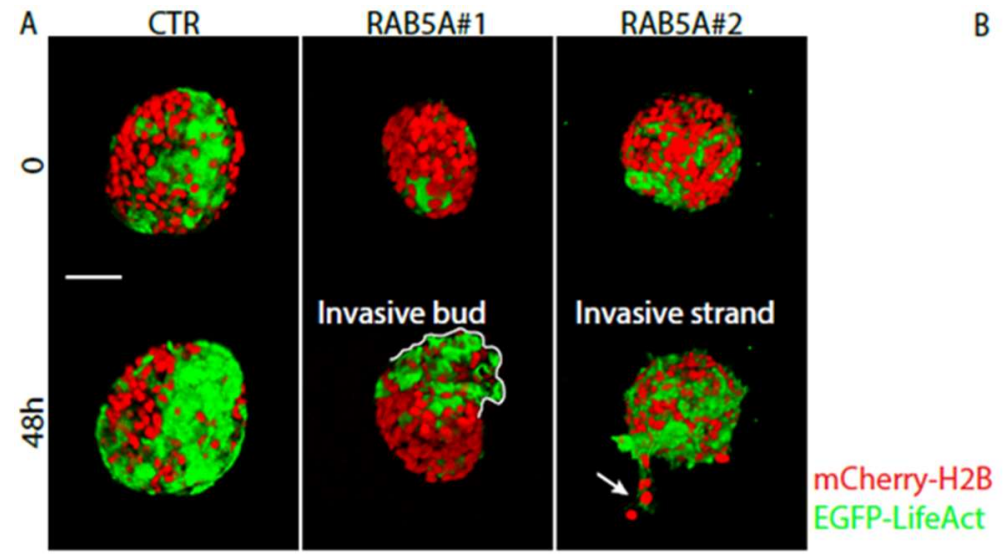

B

Figure

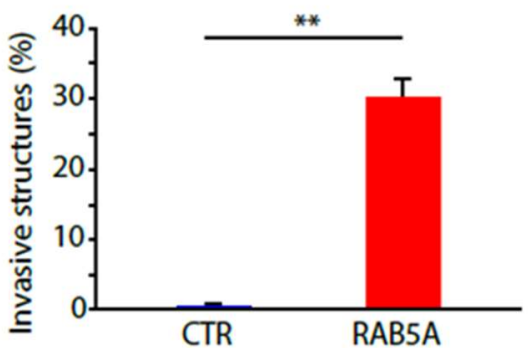

Figure 1: RAB5A promotes collective invasion in tumour spheroids

Spheroids of RAB5A-expressing tumor cells embedded in collagen type-I 3D gels form invasive structures, not observed in control spheroids. Control and RAB5A cells express EGFP-LifeAct labelling actin filaments and mCherry-H2B labelling nuclei. The histogram shows the percentage of spheroids with invasive structures expressed as the mean \pm s.d. $(n=15$ / experimental conditions in five independent experiments). 